\title{
Estimation of polarization aberrations and its effect on the point spread function of the Thirty Meter Telescope
}

Ramya M. Anche, G. C. Anupama, S. Sriram, K. Sankarasubramanian, Warren Skidmore

Ramya M. Anche, G. C. Anupama, S. Sriram, K. Sankarasubramanian, Warren Skidmore, "Estimation of polarization aberrations and its effect on the point spread function of the Thirty Meter Telescope," Proc. SPIE 10703, Adaptive Optics Systems VI, 107034K (11 July 2018); doi: 10.1117/12.2312506

EDent: SPIE Astronomical Telescopes + Instrumentation, 2018, Austin, Texas, United States 


\title{
Estimation of polarization aberrations and its effect on the point spread function of the Thirty Meter Telescope
}

\author{
Ramya M Anche ${ }^{\mathrm{a}}$, G C Anupama ${ }^{\mathrm{a}}$, S Sriramª, K Sankarasubramanian ${ }^{\mathrm{a}, \mathrm{b}}$, and Warren \\ Skidmore ${ }^{\mathrm{c}}$ \\ andian Institute of Astrophysics, Koramangala 2nd block, Bengaluru, India \\ ${ }^{\mathrm{b}}$ ISRO satellite application center, Bengaluru, India \\ 'Thirty Meter Telescope Corporation, Pasadena
}

\begin{abstract}
The Thirty Meter Telescope (TMT) is a future generation telescope proposed to be located in Mauna Kea, Hawaii or in La Palma in the Canary Islands. The telescope will have a segmented primary and an inclined tertiary mirror. The segmentation of the primary mirror and the inclination in the tertiary mirror can introduce significant polarization aberrations. Typically, the polarization aberrations, introduced due to the mirror coating and the high incident angles cause small modifications to the Point Spread Function (PSF). Here, we perform the polarization ray tracing for TMT using the optical design software Zemax for different input polarizations for a point source (on-axis). We calculate the diattenuation and retardance aberration maps for all the three mirrors of TMT. The coating induced astigmatism obtained from the retardance of the primary and secondary mirror is found to be of the order of 0.048 radians, whereas, the polarization induced tilt by the retardance of the tertiary mirror is in the order of 0.29 radians. The Jones pupil maps are estimated at two of the instrument ports, Wide Field Optical Spectrograph (WFOS) and Narrow field Infrared Adaptive Optics System (NFIRAOS).The Amplitude Response Matrix (ARM) estimated at the WFOS port show the presence of ghost PSF's. The magnitude of the ghost PSF components is of the order of $2.5 * 10^{-5}$ at $1 \mu \mathrm{m}$ at WFOS port. The ARM and the Point Spread Matrix (PSM) are estimated at the focus of the NFIRAOS instrument. The Stokes PSF is shown for horizontal and vertical polarization as inputs. The Huygen's point spread function obtained from Zemax shows the variations in FWHM for unpolarized and polarized inputs. These estimations would help in the design aspects of a high contrast imaging instrument for the TMT in the future.
\end{abstract}

Keywords: Instrumental polarization, Point spread function, Polarization aberrations, Jones Pupil map, Point Spread Matrix, Thirty Meter Telescope, NFIRAOS

\section{INTRODUCTION}

Polarization aberrations are the variations of amplitude, phase, and polarization of a wavefront across the exit pupil of an optical system. ${ }^{1}$ They are estimated using the polarization ray tracing algorithm ${ }^{2-4}$ which takes into account the changes in the polarization introduced at every interface in the system. In telescopes, these are caused due to the reflective mirror coating and the curvature of the mirrors. ${ }^{5}$ The polarization aberrations estimated for a simple Nasmyth telescope has shown the presence of coating induced astigmatism, defocus and tilt, which cannot be corrected by the adaptive optics system. ${ }^{6}$ These aberrations give rise to the ghost PSF which leads to the ellipticity of the Airy disk. Though the magnitude of these aberrations is small, they might have an effect in the high resolution and high contrast imaging instruments used in the future telescopes. ${ }^{3}$

The Thirty Meter Telescope (TMT) is one of the future generation extremely large telescopes, and will be sited either in Mauna Kea, Hawaii or La Palma in the Canary Islands. ${ }^{7}$ The optical layout as shown in Fig 1 is a Ritchey-Chretien along with the Nasmyth mirror which feeds light to various instruments on the Nasmyth platform. ${ }^{8}$ The polarization effects from the telescope optics has been estimated, which indicates that the instrumental polarization (IP) is in the order of $4-0.6 \%$ and crosstalk (CT) in the range of $70-10 \%$ in the

Further author information:

Send correspondence to Ramya M Anche, E-mail: ramyam@iiap.res.in

Adaptive Optics Systems VI, edited by Laird M. Close, Laura Schreiber,

Dirk Schmidt, Proc. of SPIE Vol. 10703, 107034K · @ 2018 SPIE

CCC code: $0277-786 \mathrm{X} / 18 / \$ 18 \cdot$ doi: $10.1117 / 12.2312506$

Proc. of SPIE Vol. 10703 107034K-1 
wavelength range of $0.45-2.5 \mu \mathrm{m} .{ }^{9,10}$ The IP and CT have been estimated at the focus of the Narrow Field Infrared Adaptive Optics System (NFIRAOS) ${ }^{11}$ (shown in Fig 2 for TMT). NFIRAOS operates in the range of 0.8-2.5 $\mu \mathrm{m}$ and feeds three instruments on the Nasmyth platform. ${ }^{12}$ NFIRAOS consists of four off-axis parabolas, two deformable mirrors and a plane mirror (field mirror) in its optical layout. ${ }^{13}$ It is necessary to estimate the polarization aberrations caused due to the three mirrors of the telescope and the optical components inside the NFIRAOS instrument to know the effects on the final point spread function.

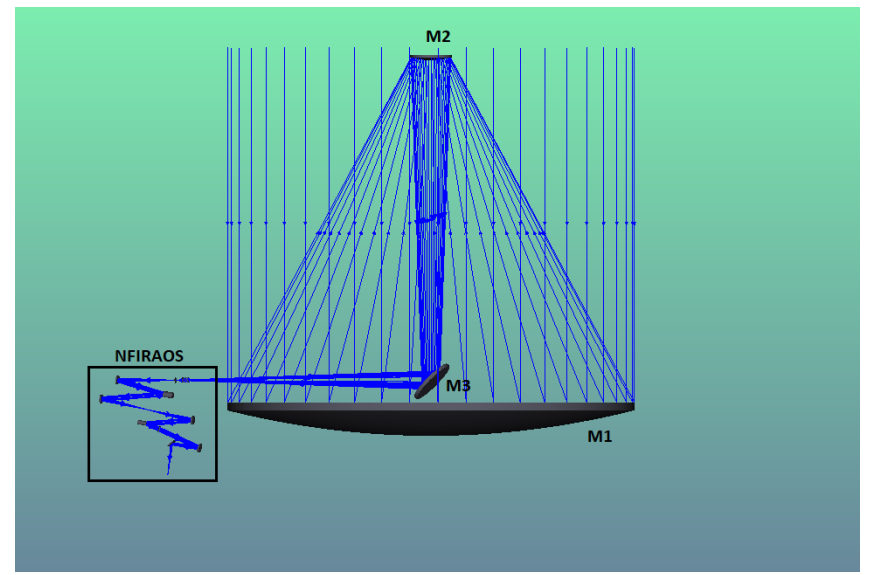

Figure 1. Optical Layout of the Thirty Meter Telescope with NFIRAOS instrument is shown. The primary mirror (M1) is a concave hyperboloid, secondary mirror (M2) is a convex hyperboloid and the Nasmyth mirror (M3) is a plane elliptical mirror.

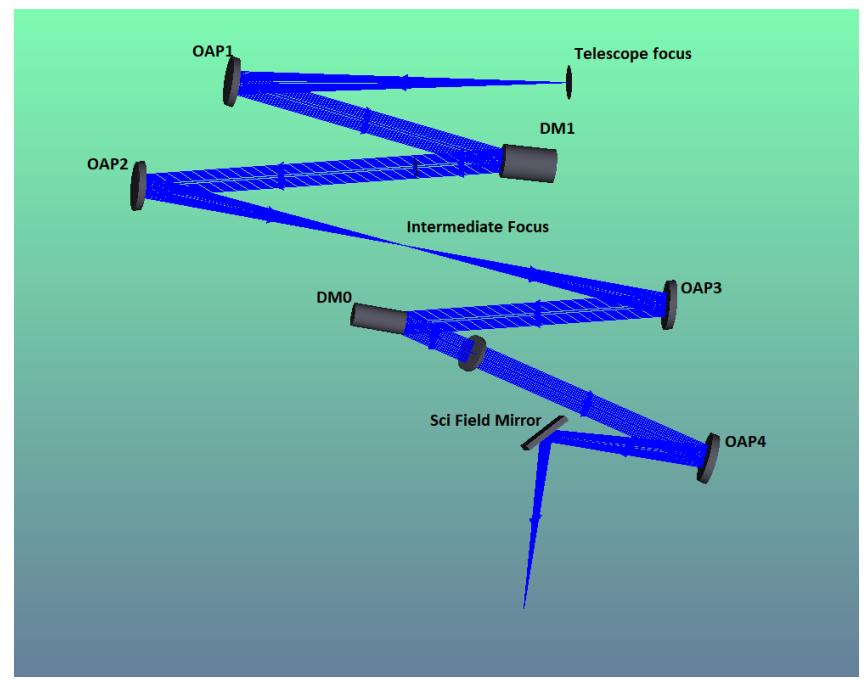

Figure 2. Optical Layout of the NFIRAOS instrument is shown in the figure. It is kept on the Nasmyth platform at an Instrument Bearing Angle(IBA)=174.5 degrees which corresponds to the tilt=-45.048 and rotation=5.499 of the Nasmyth mirror. It consists of 4 Off Axis Parabolas(OAP), 2 Deformable Mirrors (DM) and a fold mirror.

Using the polarization ray tracing in Zemax, ${ }^{14}$ we have estimated the aberrations due to the polarization from the telescope and the NFIRAOS instrument. The polarization aberration theory used here was developed by R A Chipman and James B Breckenridge. ${ }^{1,6}$

The paper is organized as follows: Section 2 explains the aberrations, diattenuation, and retardance for all the three mirrors of TMT. Jones pupil maps at Wide Field Optical Spectrograph (WFOS) port and Narrow Field Infrared Adaptive Optics System (NFIRAOS) port are given in section 3. Section 3 also has the amplitude response matrices estimated at WFOS port and NFIRAOS focus. The Point Spread Matrix (PSM) and the 
Stokes PSFs for different input polarizations are shown in section 4. The parameters estimated from PSM and Huygen's PSF from Zemax are also given in section 4. The discussion and the conclusion are given in section 5.

\section{DIATTENUATION AND RETARDANCE AT THE TELESCOPE FOCUS (WFOS PORT)}

Mirrors act as weak polarizers, as the Fresnel reflection coefficients $r_{p}$ and $r_{s}$ are not equal due to the curvature (angle of incidence) of the mirror. The polarizing capability of the mirror is represented in terms of diattenuation $\left(\mathrm{D}=\left|r_{p}\right|^{2}-\left|r_{s}\right|^{2} /\left|r_{p}\right|^{2}+\left|r_{s}\right|^{2}\right)$. The reflection coefficients have been estimated using the polarization ray tracing algorithm developed for TMT. ${ }^{10}$ The coating used for the telescope mirrors in our analysis consists of $85 \AA$ of $\mathrm{Si}_{3} N_{4}$ and $1100 \AA$ Silver. ${ }^{15}$ Fig 3 shows the diattenuation maps on the surface of primary, secondary and Nasmyth mirror of TMT. It increases quadratically from center to the edge of the mirror in the case of the primary and secondary mirror. It increases linearly in the case of Nasmyth mirror. The maximum value of diattenuation for the primary and secondary mirror is 0.000578 and 0.000694 for on axis. This indicates that the instrumental polarization (IP) introduced by the primary and secondary mirror is negligible, as expected from the symmetry properties. In the case of the Nasmyth mirror, D varies from 0.005689 to 0.007419 which is an order higher and hence the main source of the IP in the TMT.

Retardance $\left(\delta=\left|\phi_{p}-\phi_{s}\right|\right)$ corresponds to the difference in phase of the Fresnel reflection coefficients. The retardance maps for all the three mirrors are shown in Fig 4. The retardance is found to vary quadratically in the case of the primary and secondary mirror. This corresponds to the coating induced astigmatism which is one of the polarization induced aberration. The maximum retardance in the case of the primary mirror and secondary mirror is 0.022378 and 0.02686 radians respectively. The total coating induced astigmatism is 7.6 milliwaves $(\lambda / 131)$ at $1 \mu \mathrm{m}$. Retardance of the Nasmyth mirror is found to vary linearly from one end to the other end of the mirror, giving rise to polarization induced wavefront tilt. The maximum retardance for the Nasmyth mirror is 0.29524 . The corresponding tilt introduced is 0.045 waves $(\lambda / 22)$. As the Nasmyth mirror has the maximum retardance, the resultant retardance from the telescope would be dominated by it. The variation of these aberrations over the wavelength range from $0.4-2.5 \mu \mathrm{m}$ (see Table 1) indicates the presence of coating induced chromatic aberration ${ }^{5}$ caused by the dispersion of the refractive indices.

The coating induced astigmatism and tilt changes with the polarization state of the light, compared to those caused due to the surface profile of the mirror. So, the adaptive optics system will not be able to correct for these aberrations. The dependence of these aberrations on the wavelength and field would make it much more difficult to implement corrections.

\begin{tabular}{|c|c|c|c|c|c|}
\hline Wavelegth $(\mu \mathrm{m})$ & $\begin{array}{l}\text { Retardance (Rad) } \\
\text { Primary mirror }\end{array}$ & $\begin{array}{l}\text { Retardance (Rad) } \\
\text { Secondary Mirror }\end{array}$ & $\begin{array}{l}\text { Retardance (Rad) } \\
\text { Nasmyth Mirror }\end{array}$ & $\begin{array}{l}\text { Polarization induced } \\
\text { Astigmatism in waves }\end{array}$ & $\begin{array}{l}\text { Polarization induced } \\
\text { Tilt in waves }\end{array}$ \\
\hline 0.4 & 0.0805 & 0.0918 & 0.8951 & $0.0274(\lambda / 36.46)$ & $0.1425(\lambda / 7.01)$ \\
\hline 0.4592 & 0.0622 & 0.0710 & 0.7300 & $0.0212(\lambda / 47.17)$ & $0.1162(\lambda / 8.60)$ \\
\hline 0.5166 & 0.0518 & 0.0592 & 0.6247 & $0.0177(\lambda / 56.58)$ & $0.0994(\lambda / 10.05)$ \\
\hline 0.5636 & 0.0460 & 0.0525 & 0.5618 & $0.0157(\lambda / 63.75)$ & $0.0894(\lambda / 11.18)$ \\
\hline 0.6199 & 0.0408 & 0.0465 & 0.5031 & $0.0139(\lambda / 71.97)$ & $0.0801(\lambda / 12.48)$ \\
\hline 0.6526 & 0.0381 & 0.0435 & 0.4723 & $0.0130(\lambda / 77.05)$ & $0.0752(\lambda / 13.30)$ \\
\hline 0.7293 & 0.0332 & 0.0380 & 0.4158 & $0.0113(\lambda / 88.26)$ & $0.0662(\lambda / 15.11)$ \\
\hline 0.8266 & 0.0286 & 0.0327 & 0.3603 & $0.0097(\lambda / 102.33)$ & $0.0573(\lambda / 17.43)$ \\
\hline 0.9537 & 0.0244 & 0.0278 & 0.3090 & $0.0083(\lambda / 120.33)$ & $0.0492(\lambda / 20.33)$ \\
\hline 1.033 & 0.0224 & 0.0256 & 0.2844 & $0.0076(\lambda / 131.06)$ & $0.0453(\lambda / 22.09)$ \\
\hline 1.24 & 0.0184 & 0.0210 & 0.2345 & $0.0063(\lambda / 159.58)$ & $0.0373(\lambda / 26.79)$ \\
\hline 2 & 0.0124 & 0.0142 & 0.1590 & $0.0042(\lambda / 236.50)$ & $0.0253(\lambda / 39.51)$ \\
\hline 2.296 & 0.0108 & 0.0123 & 0.1385 & $0.0037(\lambda / 271.85)$ & $0.0220(\lambda / 45.37)$ \\
\hline 2.583 & 0.0096 & 0.0110 & 0.1233 & $0.0033(\lambda / 305.43)$ & $0.0196(\lambda / 50.94)$ \\
\hline
\end{tabular}

Table 1. Maximum values of the polarization-induced wavefront aberration by three mirrors of the TMT telescope at different wavelengths.

\section{JONES PUPIL MAP AND AMPLITUDE RESPONSE MATRIX}

The Jones pupil map shows the distribution of the amplitude and phase of the electric field vector in the exit pupil. It is estimated by polarization ray tracing algorithm for horizontal ( $x$-polarized) and vertical polarized ( $y$-polarized) light as inputs. Ideally, in the absence of the polarization aberrations, the Jones matrix obtained at 


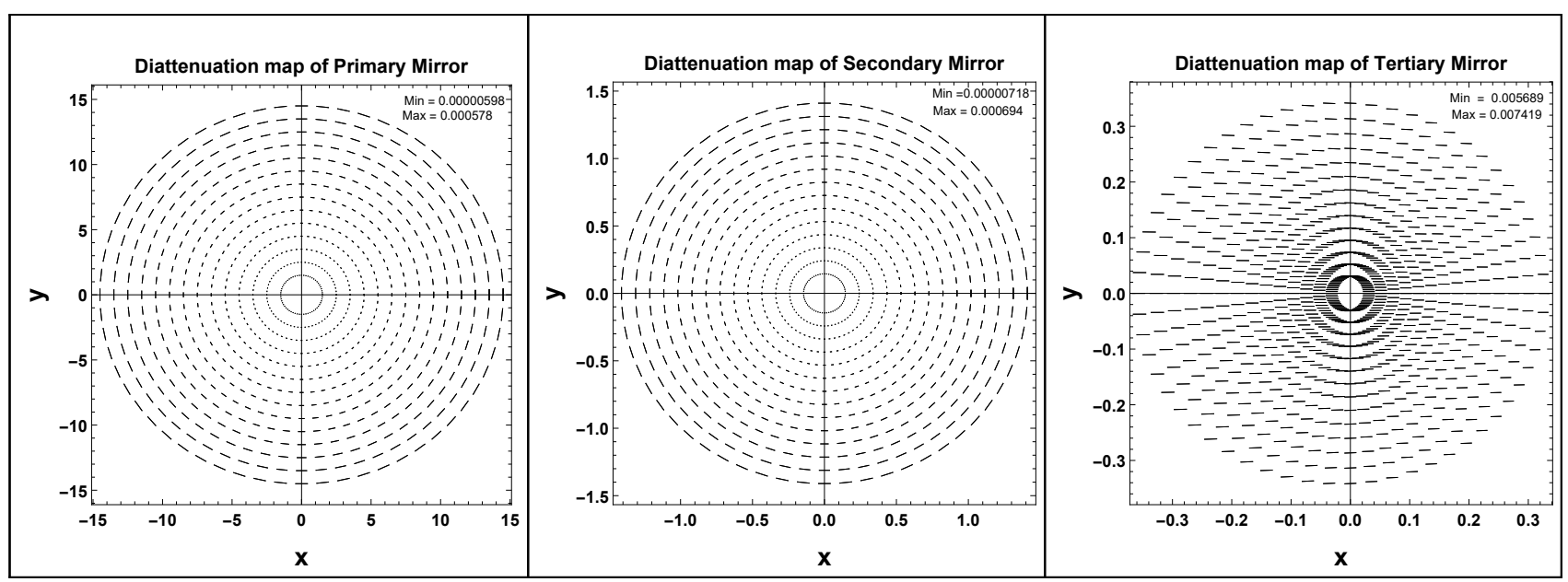

Figure 3. Diattenuation maps for all the three mirrors of the TMT is shown in the figure. The length of the line segment shows the magnitude of the diattenuation and the orientation of the segment indicates the orientation of the $\mathrm{s}$ and $\mathrm{p}$ vectors. In the case of primary and secondary mirror the diattenuation increases quadratically from center to the edge of the mirror and in the case of Nasmyth mirror, a linear increase is seen. The vertical axis in the case of Nasmyth mirror corresponds to it's tilt axis. The maximum and minimum values of the diattenuation are mentioned.
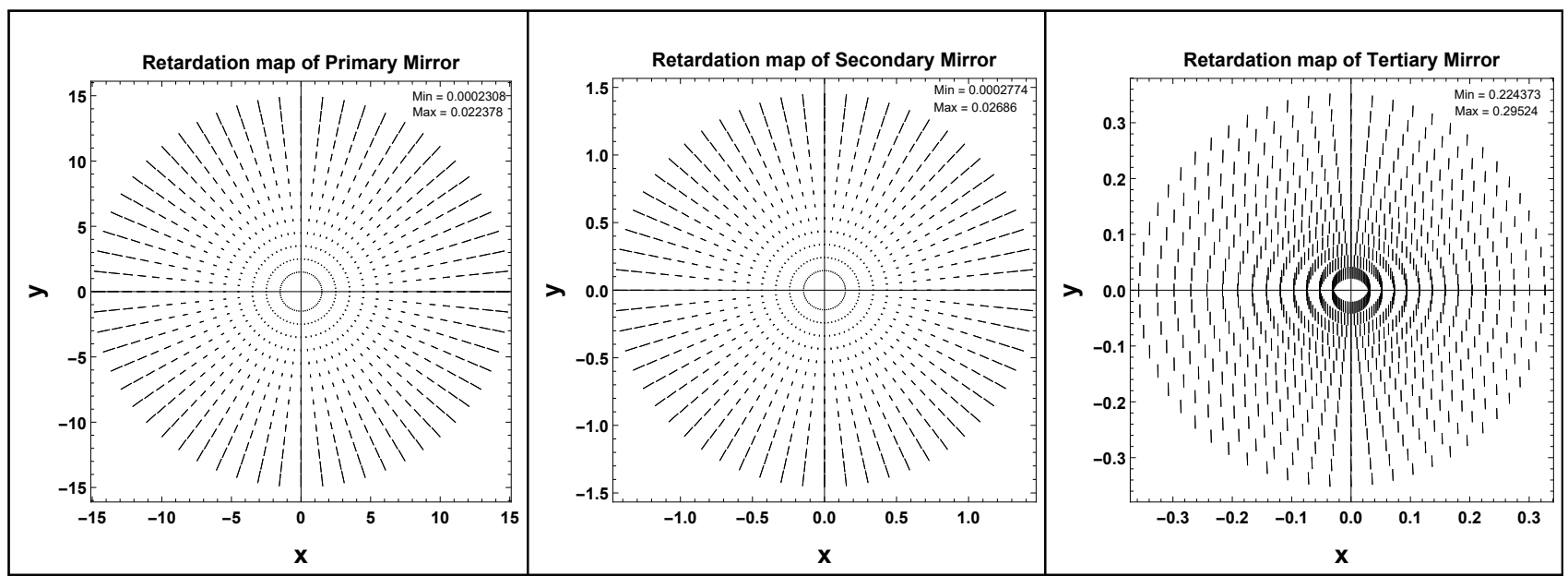

Figure 4. Retardance maps for all the three mirrors of the TMT are shown in the figure. The length of the line segment shows the magnitude of the retardance and the orientation of the segment indicates the orientation of the $\mathrm{s}$ and $\mathrm{p}$ vectors. In the case of the primary and secondary mirror, the retardance increases quadratically from center to the edge of the mirror and it increases linearly in the case of Nasmyth mirror. The vertical axis in the case of Nasmyth mirror corresponds to it's tilt axis. The maximum and minimum values of the retardance are mentioned.

the output will be an identity matrix. But due to the complex refractive indices of the coating and the curvature of the mirrors, it deviates from the ideal scenario.

\subsection{At the Wide field optical spectrograph port.}

Jones amplitude and phase pupil map given in Fig 5 are estimated at the Wide-Field Optical Spectrograph (WFOS) port of TMT. The tertiary mirror has the tilt of 45 degrees at this port. It rotates from this reference position to any other instrument port.

In the Fig 5, Axx and Ayy correspond to the output amplitude for all the pupil positions for the $x$ and $y$ polarized light at the input respectively. Axy and Ayx correspond to the cross-coupled components for $x$ and $y$ polarized light at the input. The amplitudes $A x x$ and $A y y$ at the exit pupil are not equal for $\mathrm{x}$ and y polarized light. The $x$-polarized image is found to be $1.1 \%$ brighter than the $y$-polarized one. Cross-coupled components 
Axy and Ayx show Maltese cross pattern. ${ }^{6}$ The amplitude $A x x$ and $A y y$ equal $\approx 0.92$ due to the reflection losses from the mirror coating. Axy and $A y x$ equal $\approx 0.025$, as reflection coefficients are not equal. Fig 5 also shows the phase variations $P h x x$ and Phyy at the exit pupil for $x$ and $y$ polarized light which gives the coating induced wavefront aberrations. Phxx and Phyy are not equal, which introduces the tilt and astigmatism to the incoming wavefront. Their plot looks similar to that of astigmatism and defocus aberration. Phyx and Phxy change the phase by $\pi$ radians when the amplitude becomes zero. This is similar to what was estimated in ${ }^{6}$ for a $2.4 \mathrm{~m}$ Nasmyth telescope with aluminum coating.

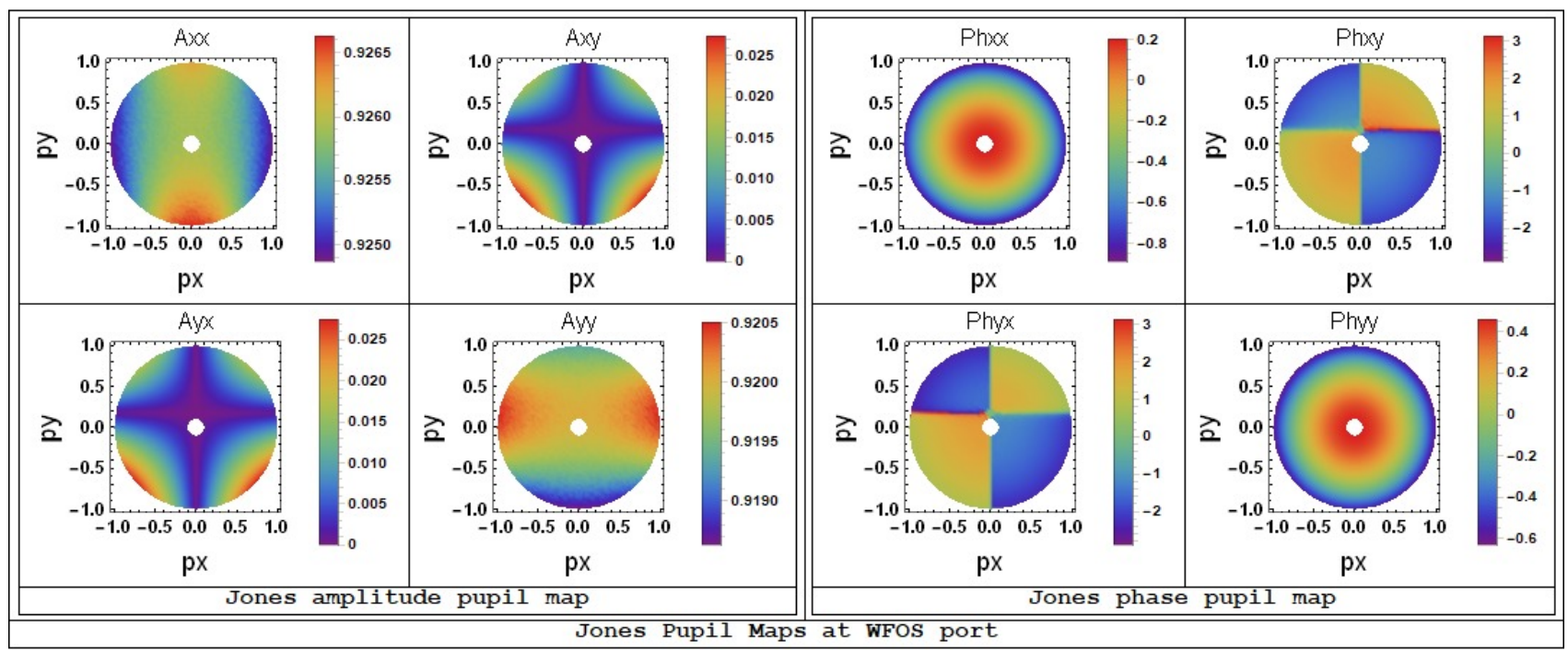

Figure 5. The Jones pupil map is estimated at the WFOS port where the M3 mirror is kept at a tilt of 45 degrees. The Jones amplitude pupil map shows the amplitude variations across the exit pupil in the TMT. Axx and Ayy show the amplitudes for $x$ and $y$ respectively. $A x y$ and $A y x$ show the amplitude of $x$ polarized light converted to $y$ polarized light and vice versa. The Jones phase pupil map shows the phase variations across the exit pupil in the TMT. Phxx and Phyy show the amplitudes for $x$ and $y$ polarized light respectively. Phxy and Phyx show the phase of $x$ polarized light converted to $y$ polarized light and vice versa. The phases for the horizontal polarization is found to be different than the vertical polarization. In the cross components, the phase changes by $\pi$ when the amplitude becomes zero.

The spatial Fourier transform over the Jones pupil elements gives the Amplitude Response Matrix (ARM), ${ }^{3}$ which is similar to the Airy disk pattern. The amplitudes for the diagonal elements are not equal due to the tilt of the Nasmyth mirror. The off-diagonal elements appear as ghost PSF's. Even though the amplitude of the ghost PSF is as small as 0.005 , it can affect during high contrast imaging using coronagraphs. They also cause an elongation of the Airy disk making it elliptical. The Amplitude response matrix at the WFOS port is given in the Fig 6 at $1 \mu \mathrm{m}$.

The diattenuation map, Jones pupil map and ARM obtained at the WFOS port clearly indicate the presence of the polarization induced wavefront aberrations. In order to understand the effect of these on the performance of the adaptive optics system of TMT (diffraction limited airy disk), we need to estimate these parameters at the NFIRAOS port and the focus of the NFIRAOS instrument which is explained in the next section.

\subsection{At the Narrow Field Infrared Adaptive Optics System (NFIRAOS) port and it's focus.}

The optical layout of NFIRAOS ${ }^{16}$ is given in the Fig 2. The Jones pupil map is estimated at the NFIRAOS port (tilt $\phi=-45.048$ and rotation, $\theta=5.4999$ ) and at the focus of the NFIRAOS. Fig 7 gives the Jones amplitude and phase maps at the NFIRAOS port. The amplitude of all the components varies from that of the WFOS port which is due to the rotation of the Nasmyth mirror. Axx and Ayy terms are flipped as the mirror is tilted in the opposite direction to what it was at the WFOS port. Axy and Ayx show the smeared Maltese cross-like pattern but the amplitude in the center is not equal to zero. The amplitude near the outer region of the pupil increases from 0.025 to 0.092 . The cross-coupled terms amount to nearly $1 \%$ of the total intensity for the $x$ and 


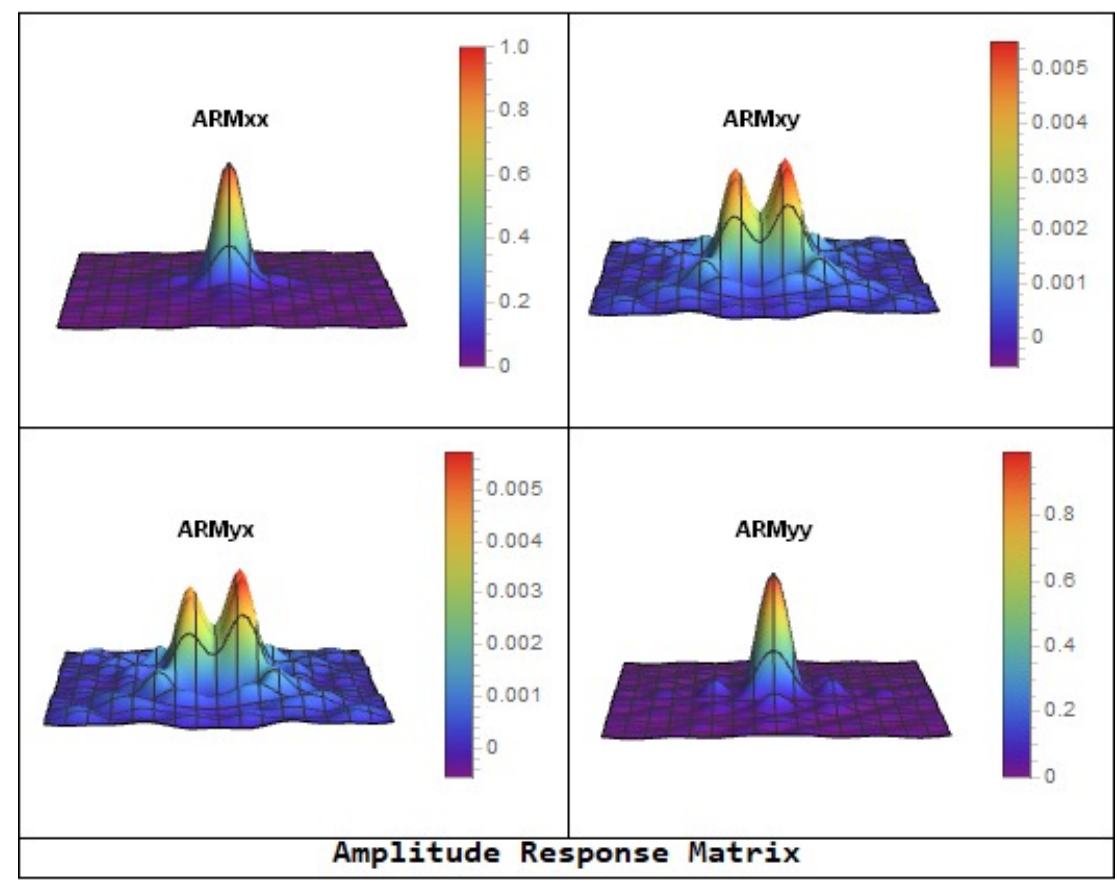

Figure 6. The Amplitude Response Matrix obtained at the WFOS port at $1 \mu \mathrm{m}$ is shown in the figure. The peaks are normalized with the maximum value of $A R M x x$.

$y$ polarized light. The Jones phase maps are shown in the Fig 7. Phxx and Phyy plots look similar to the ones obtained at the WFOS port with an increase in the magnitude. The plots of Phxy and Phyx has changed due to the rotation of the mirror thus changing the polarization aberration. Fig 8 shows the Jones amplitude and phase

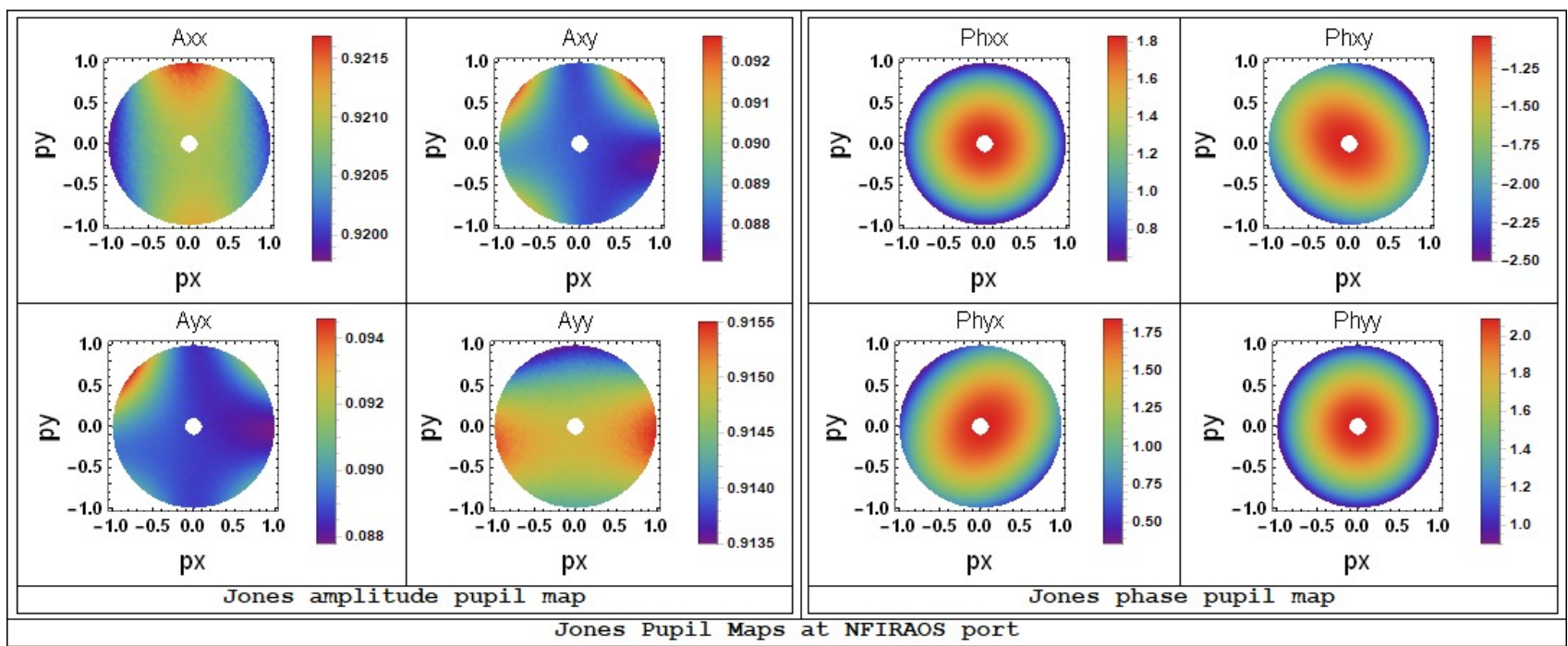

Figure 7. The Jones pupil map is estimated at the NFIRAOS port where the M3 mirror is kept at a tilt of -45.048 and rotation of 5.499 degrees. The Jones amplitude pupil map shows the amplitude variations across the exit pupil. The amplitudes for the $x$ polarization is found to be greater than the $y$ polarization. Axx and Ayy show the amplitudes for $x$ and $y$ respectively. Axy and $A y x$ show the amplitude of $x$ polarized light converted to $y$ polarized light and vice versa. The Jones phase pupil map shows the phase variations across the exit pupil. Phxx and Phyy show the phases for $x$ and $y$ polarized light respectively. Phxy and Phyx show the phases of $x$ polarized light converted to $y$ polarized light and vice versa. 
maps at the focus of NFIRAOS. They look similar to what is obtained at the NFIRAOS port. The amplitudes $A x x$ and Ayy have reduced due to further reflections from the mirrors in the instrument. The amplitudes of the cross components have also reduced compared to ones at the instrument port. The nature of the phase plots remains same as that of the NFIRAOS port indicating the instrument optics are not adding further aberrations. The coating used for all the mirrors in the NFIRAOS instrument is Silver with $\mathrm{Sio}_{2}$ as the protective layer. Further, the incident angles were estimated for the chief ray and marginal ray at all the mirror surfaces (given in Table 2). The incident angles do not vary much from the center to edge for the mirrors in NFIRAOS. So they do not add further polarization aberrations. However, the effect of the aberrations introduced by the telescope mirrors is present.

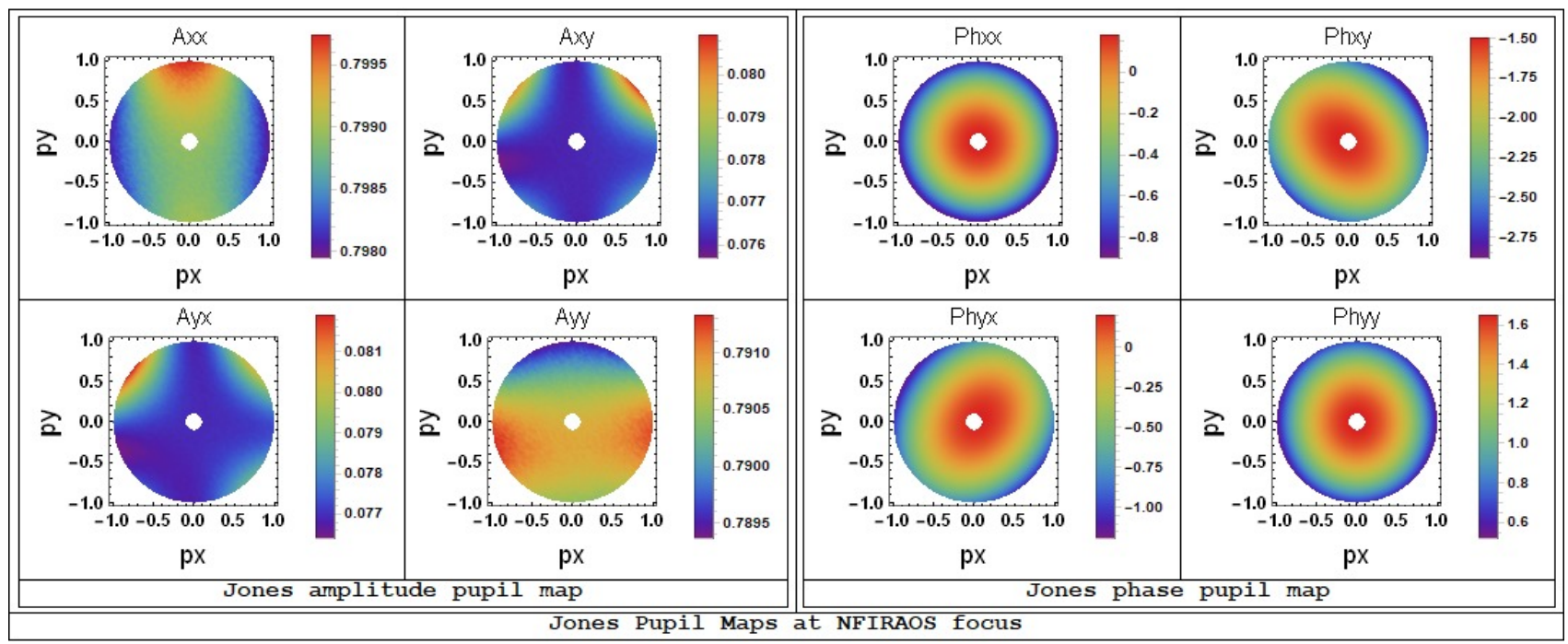

Figure 8. The Jones pupil map is estimated at the focus of the NFIRAOS instrument. The Jones amplitude pupil map shows the amplitude variations across the exit pupil. The amplitudes for the horizontal polarization is found to be greater than the vertical polarization. $A x x$ and $A y y$ show the amplitudes for $x$ and $y$ respectively. $A x y$ and $A y x$ show the amplitude of $x$ polarized light converted to $y$ polarized light and vice versa. The Jones phase pupil map shows the phase variations across the exit pupil. Phxx and Phyy show the phases for $x$ and $y$ polarized light respectively. Phxy and Phyx show the phases of $x$ polarized light converted to $y$ polarized light and vice versa.

\begin{tabular}{|c|c|c|c|c|c|}
\hline $\begin{array}{c}\text { Pupil Positions } \rightarrow \\
\text { Surfaces } \downarrow\end{array}$ & $(0,0)$ & $(1,0)$ & $(-1,0)$ & $(0,1)$ & $(0,-1)$ \\
\hline M1 & 0 & 14.0358 & 14.0358 & 14.0358 & 14.0358 \\
M2 & 0 & 14.99 & 14.99 & 14.99 & 14.99 \\
M3 & 45.0479 & 44.896 & 45.262 & 46.949 & 43.146 \\
OAP1 & 8.085 & 8.18 & 7.998 & 7.085 & 8.98 \\
DM11 & 10 & 10 & 9.9999 & 9.999 & 10 \\
OAP2 & 8.265 & 8.24 & 8.464 & 9.4384 & 7.08 \\
OAP3 & 8.2651 & 8.24 & 8.464 & 9.438 & 7.08 \\
DM0 & 13.75 & 13.74 & 13.75 & 13.75 & 13.75 \\
OAP4 & 8.036 & 8.18 & 7.999 & 7.0862 & 8.98 \\
Sci-fld mirror & 45 & 45.21 & 44.848 & 43.099 & 46.9 \\
\hline
\end{tabular}

Table 2. Incident angles at different normalized pupil positions for different mirrors in the telescope and NFIRAOS.

The amplitude response matrix obtained at the NFIRAOS focus is shown in Fig 9. ARMxx and ARMyy look similar to that obtained at the WFOS port. The cross-coupled components ARMxy and ARMyx have increased to 0.08 from 0.0025 at the WFOS port, which might cause significant effects on the final PSF. 


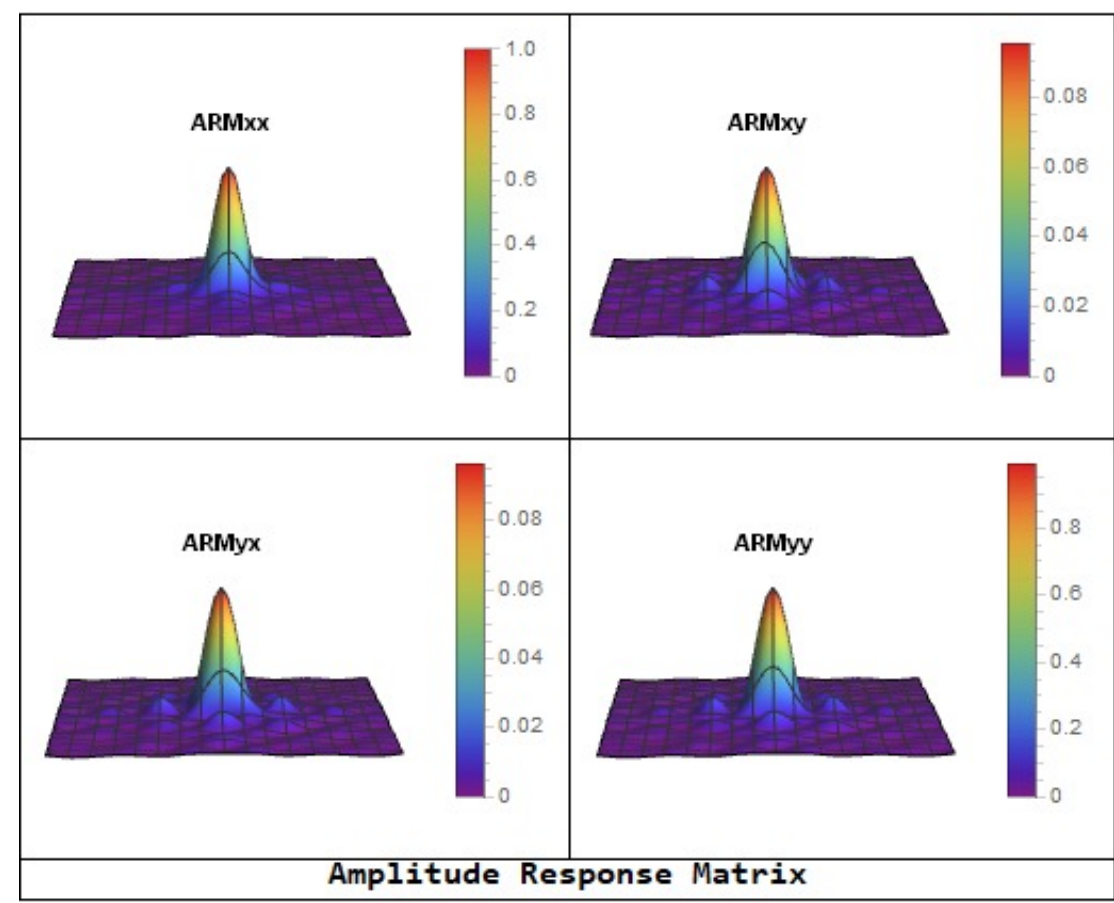

Figure 9. The Amplitude response matrix obtained at the NFIRAOS focus at $1 \mu \mathrm{m}$ is shown in the figure. The peaks are normalized with the maximum value of $A R M x x$.

\section{POINT SPREAD MATRIX (PSM) AND STOKES PSFS}

The Point Spread Matrix (PSM) is the Mueller matrix generalization of the PSF which is estimated by converting the Jones matrix (ARM here) terms into Mueller matrix terms. ${ }^{17}$ During the polarization measurements, the input Stokes vectors are multiplied with the Mueller matrix of the telescope and the instrument, to obtain output Stokes vectors. ${ }^{18}$ Stokes vectors for different input polarization are multiplied with the PSM to determine the output Stokes image components. The PSM at the NFIRAOS focus obtained by using the Jones pupil maps (shown in Fig8) is shown in Fig 10. Based on this, we estimate the output PSFs for three input polarizations.

\subsection{Unpolarized light}

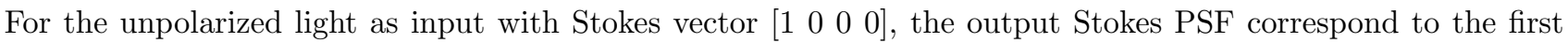
column of the PSM (in fig 10). We see $Q, U$ and $V$ components along with the intensity component $(I)$. The $Q$ and $U$ components arise due to the diattenuation of all the mirrors in the optical layout. If the diattenuation was zero then $Q$ and $U$ components would be zero as well. $V$ component which causes ellipticity is due to the fold mirrors in the optical configuration. The peak values of $Q$ and $U$ components are $1 \%$ and $0.15 \%$ respectively. $0.003 \%$ indicate smaller ellipticity in the final PSF for the unpolarized light.

\section{2 $\mathrm{X}$ and $\mathrm{Y}$ polarized light}

The $x$-polarized light or horizontally polarized light is given by the Stokes vector $\left[\begin{array}{llll}1 & 1 & 0 & 0\end{array}\right]$ and $y$ polarized or

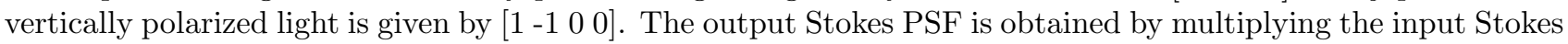
vector with the PSM. In both cases, we see $U$ and $V$ components due to the diattenuation and retardance of the mirrors in the optical layout. The effect of light coupled into the orthogonal components has to be estimated. Fig 11 shows the Stokes PSF image for the $x$ polarized light. We see the peak value of the $U$ component as 0.15 and $V$ component as 0.00050 . Fig 12 shows the Stokes PSF images for the $y$ polarization as input. The peak value of $U$ is -0.15 and $V$ is -0.00075 . Using these PSFs, we estimate the final intensity profiles for the polarized states. The total irradiance for $x$ and $y$-polarized light would contain the effect of the crossed component also. 


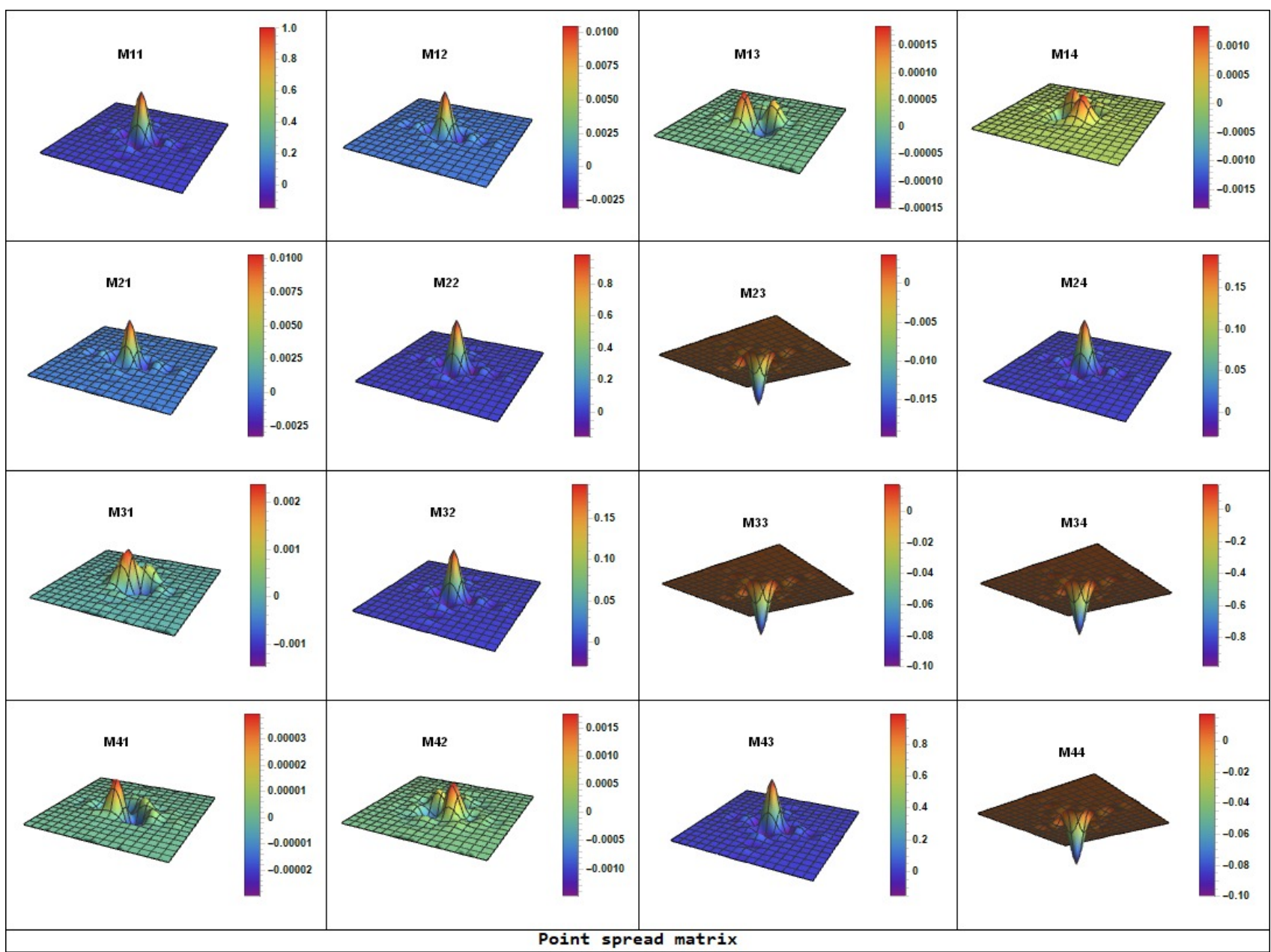

Figure 10. The point spread matrix estimated at the NFIRAOS focus at the wavelength of $1 \mu \mathrm{m}$. The input Stokes vectors are multiplied with this matrix to obtain the corresponding Stokes PSF at the instrument focus.

$I x=I x x+I y x$ and $I y=I y y+I x y$ where Ix corresponds to the PSF for $X$-polarized light and $I y$ is the PSF for the $Y$-polarized light. Ixx, Iyx, Iyy and Ixy can be estimated from the PSM as follows: ${ }^{6}$

$$
I x x=\frac{I x+Q x}{2} ; \quad \text { Iyx }=\frac{I x-Q x}{2} ; \text { Iyy }=\frac{I x-Q x}{2} ; \quad \text { Iyy }=\frac{I x+Q x}{2}
$$

After estimating $I x$ and $I y$ using the Stokes PSFs, we consider cross-sections through the maxima and plot corresponding $I x$ and $I y$ and their difference is shown in the Fig 13. Ix and $I y$ are not equal and are not exactly superposed over the other. This is caused due to the difference in the phases of the reflection coefficients. The following parameters are estimated from the final PSF obtained using PSM for X and Y polarized light.

1. Comparison of the total fluxes.

$$
\frac{\text { Flux Iyx }}{\text { Flux Ixx }}=0.940024 \% ; \quad \frac{\text { Flux Iyy }}{\text { Flux Ixx }}=97.9668 \% ; \frac{\text { Flux Ixy }}{\text { Flux Ixx }}=0.94064 \% ;
$$

2. Comparison of the peak values.

$$
\frac{\text { Peak Iy }}{\text { Peak Ix }}=97.9266 \% ; \quad \frac{\text { Peak }(I x-I y)}{\text { Peak Ix }}=2.0738 \%
$$




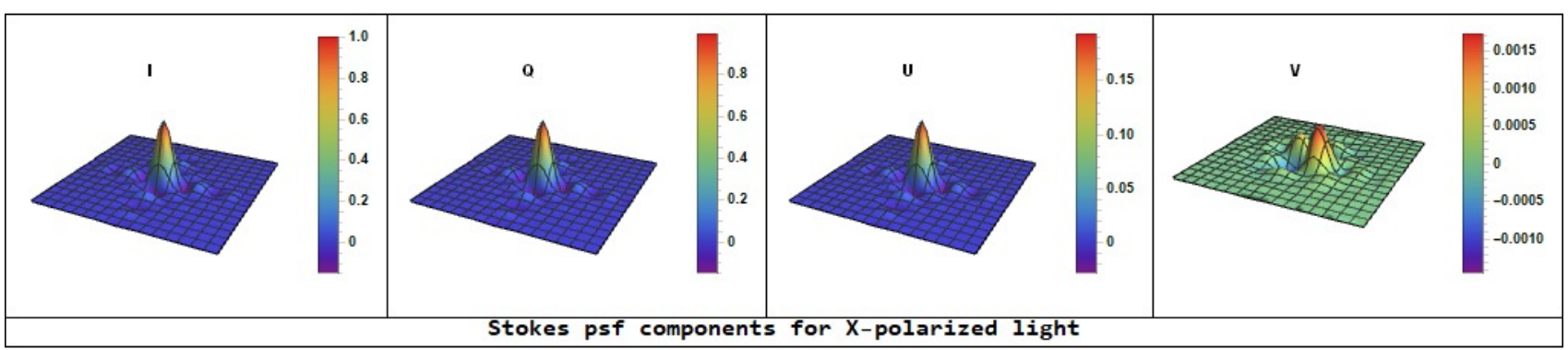

Figure 11. The Stokes PSF components estimated at the NFIRAOS focus for the $x$-polarized light as input. It is estimated at a wavelength of $1 \mu \mathrm{m}$.

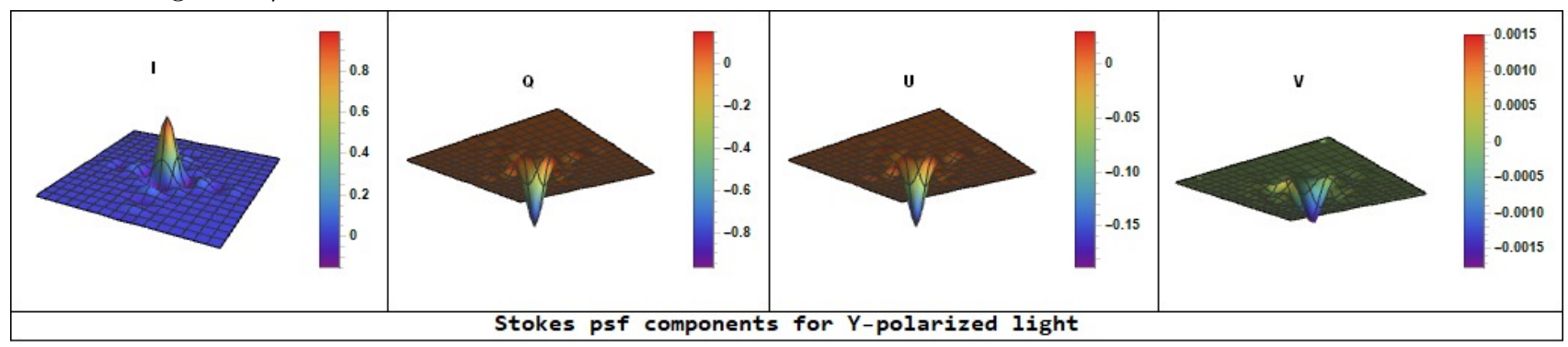

Figure 12. The Stokes PSF components estimated at the NFIRAOS focus for the $y$-polarized light as input. It is estimated at the wavelength of $1 \mu \mathrm{m}$.

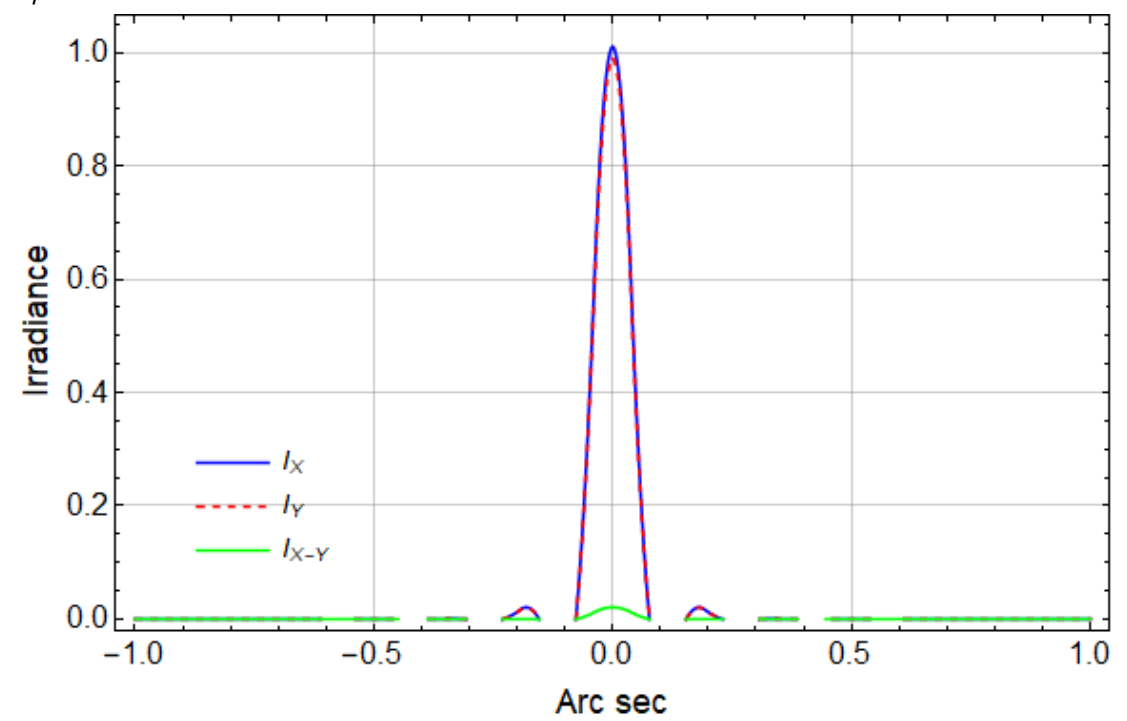

Figure 13. The cross section of $I x$ and Iy PSF images is shown in the figure. The difference in the intensities between two polarized PSFs is also shown in green color.

\subsection{Effect on the final PSF}

We have obtained the Huygen's PSF using Zemax, considering the polarization aberrations to estimate the shear, FWHM and ellipticity in the final PSF at the NFIRAOS focus. The $X$ and $Y$ cross-sections of the PSF are considered for each case to estimate the ellipticity.

1. Unpolarized light

$$
\begin{array}{r}
F W H M(X-\text { direction })=6.98 \pm 0.032 \text { mas } \\
F W H M(Y-\text { direction })=6.94 \pm 0.032 \text { mas } \\
\text { Ellipticity of the PSF }=5.730 * 10^{-3}
\end{array}
$$


2. $X$-polarized light

$$
\begin{array}{r}
F W H M(X-\text { direction })=7.025 \pm 0.032 \text { mas } \\
F W H M(Y-\text { direction })=6.93 \pm 0.032 \text { mas } \\
\text { Ellipticity of the PSF }=0.01352
\end{array}
$$

3. $Y$-polarized light

$$
\begin{array}{r}
F W H M(X-\text { direction })=6.98 \pm 0.035 \text { mas } \\
F W H M(Y-\text { direction })=6.96 \pm 0.032 \text { mas } \\
\text { Ellipticity of the PSF }=2.865 * 10^{-3}
\end{array}
$$

4. Shear between $X$ and $Y$ polarizations $=0.045 \mathrm{mas}$

\section{DISCUSSIONS AND CONCLUSIONS}

1. The polarization aberrations in the TMT are coating induced astigmatism, tilt and chromatic aberration. These are caused by the mirror coating, curvature of the primary and secondary mirrors, and the tilt of the Nasmyth mirror. The diattenuation and retardance maps are shown for all the three mirrors of the telescope.

2. Jones pupil maps are obtained at two instrument ports and focus of the NFIRAOS instrument. The rotation of the Nasmyth mirror is found to cause changes in the map. The optical components in the NFIRAOS instruments are found to cause little or no effect on the map obtained at its port.

3. The amplitude response matrix obtained at the WFOS port shows the presence of the ghost PSF of amplitude 0.005 in the cross-components. At the NFIRAOS port, the amplitude of the cross-components increase to 0.08 .

4. The point spread matrix obtained by Jones-Mueller conversion gives the Stokes output PSFs for any input Stokes vector. It shows that the PSF of an unpolarized source does not remain unpolarized at the NFIRAOS focus. The $x$ and $y$ polarized light show the presence of $V$ component which causes the ellipticity of the resultant PSF.

5. The Huygen's PSF estimated from Zemax shows the variations in the FWHM for different input polarizations.

6. Adaptive optics system cannot correct for the aberrations in both the polarizations $(X$ and $Y)$ simultaneously. This may affect in the future generation instrument with coronograph for the TMT. The intensities obtained can be considered during the design of the high contrast imaging instrument.

7. To estimate the impact of polarization aberrations on final Strehl ratio, the near diffraction limited PSF obtained from the adaptive optics system has to be convolved with the Stokes PSF.

\section{ACKNOWLEDGMENTS}

Authors would like to thank Prof. Jayant Murthy and Mr. Joice Mathew of Indian Institute of Astrophysics for the Zemax software license. 


\section{REFERENCES}

[1] Chipman, R. A., "Polarization aberrations (thin films).," (1987).

[2] Chipman, R. A. and Lam, W. S. T., "The polaris-m ray tracing program," in [Polarization Science and Remote Sensing VII], 9613, 96130J, International Society for Optics and Photonics (2015).

[3] Chipman, R. A., Lam, W. S. T., and Breckinridge, J., "Polarization aberration in astronomical telescopes," in [Polarization Science and Remote Sensing VII], 9613, 96130H, International Society for Optics and Photonics (2015).

[4] Chipman, R. A., "Mechanics of polarization ray tracing," Optical Engineering 34(6), 1636-1646 (1995).

[5] Reiley, D. J. and Chipman, R. A., "Coating-induced wavefront aberrations," in [Polarization Analysis and Measurement], 1746, 139-147, International Society for Optics and Photonics (1992).

[6] Breckinridge, J. B., Lam, W. S. T., and Chipman, R. A., "Polarization aberrations in astronomical telescopes: the point spread function," Publications of the Astronomical Society of the Pacific 127(951), 445 (2015).

[7] Sanders, G. H., "The thirty meter telescope (tmt): An international observatory," Journal of Astrophysics and Astronomy 34(2), 81-86 (2013).

[8] TMTGroup, "Design requirements document for tertiary mirror system m3s," (2012).

[9] Anche, R. M., Sen, A. K., Anupama, G. C., Sankarasubramanian, K., and Skidmore, W., "Analysis of polarization introduced due to the telescope optics of the thirty meter telescope," Journal of Astronomical Telescopes, Instruments, and Systems 4(1), 018003 (2018).

[10] Anche, R. M., Anupama, G., Reddy, K., Sen, A., Sankarasubramanian, K., Ramaprakash, A., Sengupta, S., Skidmore, W., Atwood, J., Tirupathi, S., et al., "Analytical modelling of thirty meter telescope optics polarization," in [International Conference on Optics and Photonics 2015], 9654, 965408, International Society for Optics and Photonics (2015).

[11] Atwood, J., Skidmore, W., Anupama, G., Anche, R. M., Reddy, K., and Sen, A., "Polarimetric analysis of the thirty meter telescope (tmt) for modeling instrumental polarization characteristics," in [Modeling, Systems Engineering, and Project Management for Astronomy VI], 9150, 915013, International Society for Optics and Photonics (2014).

[12] Herriot, G., Andersen, D., Atwood, J., Byrnes, P., Boucher, M.-A., Boyer, C., Caputa, K., Correia, C., Dunn, J., Ellerbroek, B., et al., "Tmt nfiraos: adaptive optics system for the thirty meter telescope," in [Adaptive Optics Systems III], 8447, 84471M, International Society for Optics and Photonics (2012).

[13] Herriot, G., Andersen, D., Atwood, J., Boyer, C., Byrnes, P., Conan, R., Ellerbroek, B., Gilles, L., Hickson, P., Jackson, K., et al., "Nfiraos-first light adaptive optics system for tmt," in [1st AO4ELT conferenceAdaptive Optics for Extremely Large Telescopes], 02004, EDP Sciences (2010).

[14] Anche, R. M., Anupama, G., and Sankarasubramanian, K., "Preliminary design techniques to mitigate the polarization effects due to telescope optics of the thirty meter telescope (tmt)," Journal of Optics , 1-8 (2017).

[15] Vucina, T., Boccas, M., Araya, C., and Ahhee, C., "Gemini's protected silver coatings: first two years in operation," in [SPIE Astronomical Telescopes+ Instrumentation], 62730W-62730W, International Society for Optics and Photonics (2006).

[16] Atwood, J., Byrnes, P., and Herriot, G., "Nfiraos: the optical design of an adaptive optics system for the thirty meter telescope," in [Astronomical and Space Optical Systems], 7439, 74390G, International Society for Optics and Photonics (2009).

[17] Goldstein, D. H., [Polarized Light, revised and expanded], CRC press (2003).

[18] Tinbergen, J., [Astronomical polarimetry], Cambridge University Press (2005). 